None of the patients selected from those showing inhibited leucocyte migration with Hodgkin's spleen extract showed inhibited migration when tested with normal spleen extract. In the doseresponse experiments the normal leucocytes did not show inhibited migration at any concentration of spleen extract. The leucocytes from patients with lymphoma showed no significant migration inhibition with concentrations of less than $5 \mathrm{mg} / \mathrm{l}$. At $10 \mathrm{mg} / \mathrm{l}$ significant inhibition occurred but was not further enhanced in higher concentrates of extract (up to $200 \mathrm{mg} / \mathrm{l}$ ). Inhibition of migration with Candida albicans or PPD, or both, was seen in $35(37 \%)$ of the 94 patients with lymphoma but there was no significant correlation between the migration indices with these antigens and those with Hodgkin's spleen extract.

Skin testing gave an impaired response in $20(21 \%)$ of the 94 patients with lymphoma. There was no correlation between this assessment of cellular immunocompetence and reactivity to Hodgkin's spleen extract.

\section{Discussion}

The finding of a splenic factor that inhibits leucocyte migration in patients with lymphoma was not unexpected since other workers have shown possible antigens in non-cultured and cultured Hodgkin's disease tissues. Eshhar et $a l^{5}$ confirmed that the antigen that they showed using immunofluorescence and absorbed tumour antisera in non-cultured spleen was normal tissue ferritin. High serum ferritin levels have been found in patients with leukaemia and Hodgkin's disease ${ }^{6}$ and other neoplasms but may often be related to the non-specific anaemia of neoplastic disease with its associated increase in reticuloendothelial iron stores and decreased serum iron concentration. ${ }^{7}$ Increased ferritin synthesis by neoplastic tissue has been shown in acute leukaemia, ${ }^{8}$ but probably reflects an overall increase in protein synthesis rather than tumour cell overproduction of a specific ferritin.

The nature of the Hodgkin's disease tissue culture antigen of Long et $a l^{2} 910$ has not been established. It is not ferritin but could be a viral component, a tumour or fetal antigen, or a normal tissue factor.

Using the leucocyte migration inhibition technique we have shown sensitisation to a factor present in the spleen of a patient with Hodgkin's disease in over half of the patients with lymphoma who were tested. Sensitisation was seen much less often in other cancer patients, in other inpatients, and in normal people. Sensitisation to normal spleen tissue was not observed and there was no correlation of Hodgkin's splenic factor sensitisation with either Candida albicans or PPD sensitisation, or both, or with cellular immunocompetence. We are now trying to isolate and identify the factor, which may prove to be a helpful diagnostic tool in patients with suspected lymphoma. Its potential prognostic importance and use for detecting disease activity are also being assessed.

We thank Drs C W Potter and I A Carr for helpful criticism, the consultant staff of Weston Park Hospital for access to patients under their care, and the Cancer Research Campaign (Yorkshire Branch) for financial assistance.

\section{References}

1 Order, S E, Porter, M, and Hellman, S, New England fournal of Medicine, 1971, 285, 471.

${ }^{2}$ Long, J C, et al, Proceedings of the National Academy of Sciences of the United States of America, 1973, 70, 1540.

${ }^{3}$ Lancet, 1975, 1, 556.

4 Søberg, M, and Bendixen, G, Acta Medica Scandinavica, 1967, 181, 247.

5 Eshhar, Z, Order, S E, and Katz, D H, Proceedings of the National Academy of Science of the United States of America, 1974, 71, 3956.

${ }^{6}$ Jones, P A E, et al, British fournal of Cancer, 1973, 27, 212.

7 Jacobs, A, and Worwood, M, New England Fournal of Medicine, 1975, 292, 951.

8 White, G P, Worwood, M, and Parry, D H, Nature, 1974, 250, 584.

${ }^{9}$ Long, J C, Aisenberg, A C, and Zamecnik, P C, Proceedings of the National Academy of Sciences of the United States of America, 1974, 71, 2285.

10 Long, I C, Aisenberg, A C, and Zamecnik, P C, Proceedings of the National Academy of Sciences of the United States of America, 1974, 71, 2605.

\title{
Detection of somatic muscle fasciculation on electrocardiograms
}

\author{
D J THOMAS, D O WILLIAMS
}

British Medical fournal, 1976, 1, 557-559

\section{Summary}

Small discrete spikes appearing on the electrocardiogram (ECG) of a man with a pacemaker turned out to be fasciculation potentials picked up from leg muscles. To find out how common these spikes were all routine ECGs made in one month were reviewed. Spike potentials were found in six patients, and in each case they originated from somatic musculature. Spikes could nearly always be detected on ECGs that were recorded subsequently in patients known to have lower motor neurone lesions that produced fasciculation. Thus the presence

Queen Elizabeth Hospital, Birmingham B15 2TH

D J THOMAS, MB, MRCP, senior registrar in neurology

D O WILLIAMS, MB, MRCP, honorary senior registrar in cardiology of these fasciculation spikes may suggest underlying neuromuscular disease; this possibility should be recognised by those who record ECGs so that the spikes are not suppressed by overuse of the filter.

\section{Introduction}

There are three common causes of electrocardiographic (ECG) interference: alternating current artefact if earthing is inadequate; unstable baseline if electrode contact is poor; and "muscle noise" if the patient is cold, not relaxed, or has a tremor. Our interest was aroused by another form of interference which complicated the management of a patient with an indwelling pacemaker. This patient has been reported fully elsewhere; ${ }^{1}$ briefly, he was suffering from paroxysms of dyspnoea and palpitations, and his ECG showed sharp discrete spikes resembling those reported in a case of "runaway pacemaker." $\mathrm{We}$ therefore assumed that the spikes originated from the pacemaker, but they persisted after its replacement. We eventually discovered that they were fasciculation potentials picked up from the man's left leg muscles. 
It is not generally appreciated that somatic muscle fasciculation can be detected as spikes on the ECG. We undertook this study to determine whether these spikes were rare, depending perhaps on the presence of particularly large, superficially placed, fasciculation potentials.

\section{Patients and methods}

To assess the rarity of these fasciculation potentials we reviewed all the routine ECGs performed in this hospital for one month. These were all recorded by the technicians in the standard way using plate electrodes.

Four of the patients found to have these abnormal spikes on an ECG were studied in detail. A full neurological assessment included: history, examination, electromyography (EMG), nerve conduction studies, plain $x$-ray examination of the relevant spinal areas, and, in two cases, myelography. Their standard ECG leads 1, 2, and 3 were recorded simultaneously. The electrode from one of the normal limbs was then repositioned over an area of the limb exhibiting fasciculation (see fig 1). The binole from the two electrodes on this abnormal limb thus represented a surface EMG recorded across fasciculating muscle.

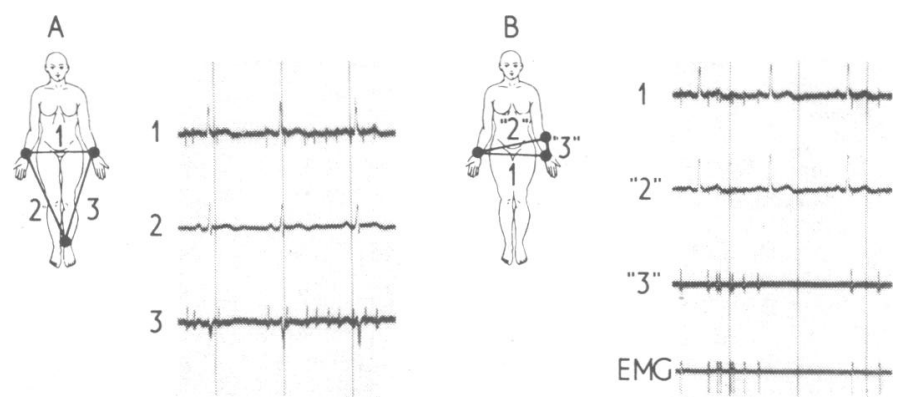

FIG 1 -Case 3. (A) Standard lead arrangement. Spikes visible in leads 1 and 3 suggest fasciculation in left arm. (B) Modified leads " 2 " and " 3 ." Lead " 3 " is surface EMG and below it is synchronous intramuscular EMG from left forearm.

Having identified the site at which the spikes were recorded by the mobile surface electrode, we obtained a simultaneous EMG by inserting a concentric needle electrode at this point. The characteristics of the abnormal potentials were studied electromyographically on a Medelec MS6.

\section{Results}

Five-hundred-and-fifty ECGs were performed in this hospital during the month sampled, and those of six patients showed spike potentials. Three had been admitted through casualty to medical wards, and the others were in hospital for cold investigation, one in the cardiological unit and two in the neurological unit. Four patients were studied in detail.

Case 1-A 61-year-old man with an indwelling demand pacemaker had diabetes mellitus managed by diet and metformin. In addition to a mild diabetic sensory neuropathy, he had dysarthria, generalised wasting, and weakness judged to be due to anterior horn cell damage.

Case 2-A 53-year-old toolsetter was admitted with hypertension. He had had poliomyelitis as a boy and had been aware of intermittent "wriggling sensations" in his muscles for many years.

Case 3-A 63-year-old labourer had severe cervical spondylosis at several levels, which produced considerable wasting and weakness of his left arm musculature.

Case 4-A 57-year-old farmer had severe backache of sudden onset and pronounced wasting and fasciculation of his right leg. Myelography showed a large prolapsed intervertebral disc at L2-L3 on the right, which was confirmed at laminectomy.

In each case studied there was no doubt that the spikes on the ECG originated from fasciculation in somatic musculature. Fig 1 shows that the ECG spikes and the surface EMG and needle EMG potentials were perfectly synchronous.

The characteristics of the fasciculation potentials in the different patients on needle EMG were not remarkable. Their amplitude varied from $200 \mu \mathrm{V}$ to $2 \mathrm{MV}$. They were either diphasic or triphasic. Their duration was also in the normal range. Most of them fired intermittently and irregularly, but others were quite regular. The rate of firing varied.

Concentric needle electrodes have a more limited range of pick-up than surface ones. This is exemplified in fig 2, where the surface recording on channel 3 shows not only many potentials synchronous with those of the needle electrode on channel 4 but also many others from denervating motor units beyond the needle's reach.
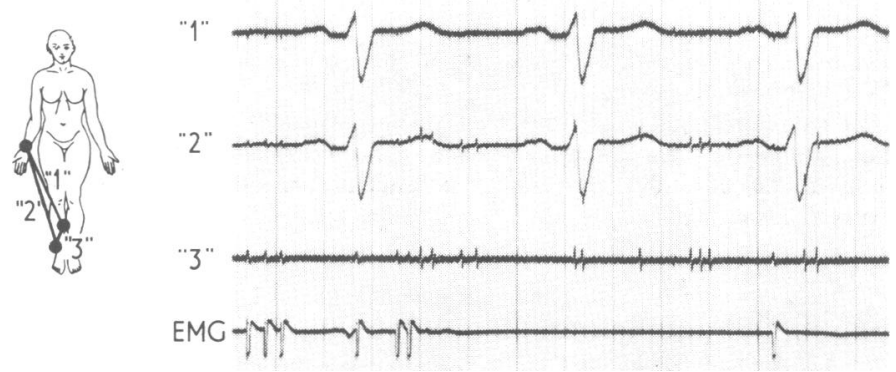

FIG 2-Case 4. Modified lead " 3 " represents surface EMG that is recording more potentials than the underlying intramuscular, concentric needle electrode on channel 4 .

Small repetitive movements, like tapping a finger or moving a toe during ECG recording, did not produce these discrete spikes, but only short bursts of "muscle noise" (fig 3). This irregular distortion of the baseline was produced by the summation of numerous potentials when recruited on voluntary contraction and is known in electromyography as the recruitment or interference pattern.

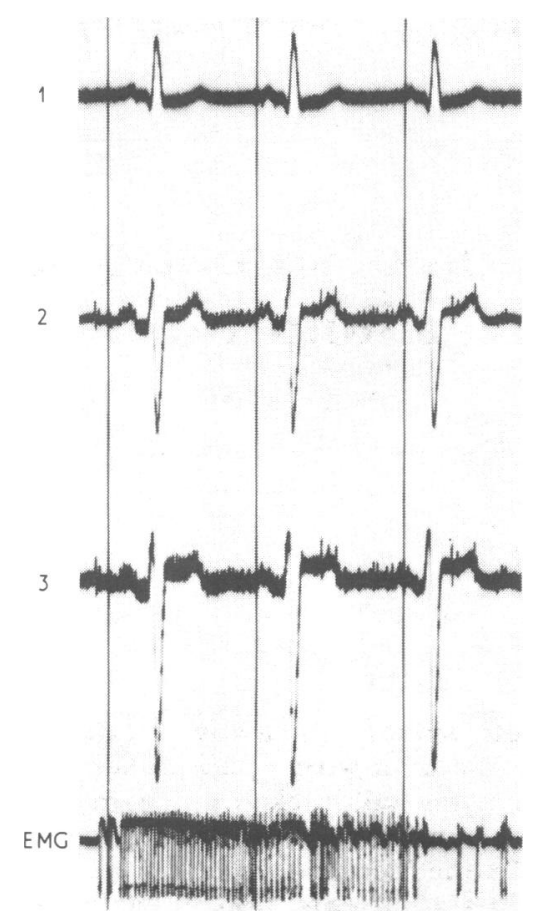

FIG 3-Case 2. "Muscle noise" on ECG leads 2 and 3 produced by twitching left big toe, with synchronous short burst of interference pattern on EMG (channel 4).

We then tried to record spike potentials by performing ECGs in other patients known to have lower motor neurone lesions producing fasciculation. We could nearly always detect the spikes on the ECG. If they were absent with electrodes in the standard positions moving a surface electrode over a muscle known to have been fasciculating usually showed them. We could often suppress the spikes so that they were no longer noticeable by generous use of the ECG recorder's filter. 


\section{Discussion}

We have shown that picking up fasciculation spikes from denervated muscle on the ECG is not a rare event. The fasciculations that can be so recorded do not have to be very large in amplitude or particularly close to the surface electrodes. Perhaps the spikes have not been emphasised before because their significance has not been recognised and therefore they have been overlooked or simply discounted.

When an ECG shows these spikes it should prompt a search for underlying neuromuscular disease, if this is not already appreciated, and attention should be drawn to them in the ECG report. It should be possible to say from which limb the dis- turbance has come by seeing in which leads the artefact occurs. The significance of these spikes should be appreciated by all those who read and record ECGs, especially the latter because by too generous a use of the filter they may be missed.

Requests for reprints should be addressed to Dr D J Thomas, The National Hospital, Queen Square, London WC1N 3BG.

\section{References}

1 Williams, D O, and Thomas, D J, British Heart fournal, in press.

${ }^{2}$ Harper, R, et al, British Heart fournal, 1974, 36, 610.

\title{
Failure of phenobarbitone to prevent febrile convulsions
}

\author{
J Z HECKMATT, A B HOUSTON, D J CLOW, J B P STEPHENSON, K L DODD, \\ G T LEALMAN, R W LOGAN
}

British Medical fournal, 1976, 1, 559-561

\section{Summary}

One-hundred-and-sixty-five children without known neurological disorder who presented with their first febrile convulsion between the ages of six months and three years were assigned to daily phenobarbitone treatment or to a control group and followed up at a special clinic for six months. One-hundred-and-sixty-one children completed the trial, and of the 88 children assigned to phenobarbitone treatment 10 had further convulsions during this period compared with 14 of the 73 control children. Only 49 of those assigned to phenobarbitone took the drug regularly throughout the trial, and four of these had further febrile convulsions, a proportion not significantly different from that in the controls. All four had mean plasma phenobarbitone concentrations over $69 \mu \mathrm{mol} / 1(16 \mu \mathrm{g} / \mathrm{ml})$ during the trial and in three the plasma concentration was at or over this figure within eight hours of the repeat convulsion. Regular phenobarbitone does not seem to prevent febrile convulsions. Attention should instead be directed to organising emergency services to allow early termination of febrile convulsions, whether first or subsequent, to prevent irreversible brain damage.

\section{Introduction}

There is now considerable evidence that febrile convulsions if prolonged and severe may cause irreversible cerebral injury,

Royal Hospital for Sick Children, Yorkhill, Glasgow

J Z HECKMATT, MB, MRCP, medical registrar

A B HOUSTON, MB, MRCP, senior medical registrar

D J CLOW, MRCP, $\mathrm{DCH}$, senior medical registrar (now consultant paediatrician, Dumfries and Galloway Royal Infirmary, Dumfries)

J B P STEPHENSON, MRCP, DCH, consultant in paediatric neurology

Departments of Child Health and Biochemistry, University of Glasgow, Glasgow

K L DODD, MRCP, DCH, senior registrar

G T LEALMAN, MRCP, DCH, registrar (now senior registrar, Bradford Children's Hospital, Bradford)

$R$ W LOGAN, MRCPATH, MRCP GLAS, consultant in biochemistry

leading to mental handicap, cerebral palsy, or secondary epilepsy, including that arising from mesial temporal sclerosis. ${ }^{1-6}$ There is also evidence that the convulsions that induce this brain damage differ from more common benign febrile convulsions only in their duration. ${ }^{2}{ }^{4}{ }^{5}$ Since the liability to prolonged convulsions increases with each recurrence ${ }^{1}$ a treatment that prevented recurrence of convulsions would diminish the number of people handicapped as a result of severe attacks. Much enthusiasm therefore greeted the publication of a controlled trial by Faerø et al, ${ }^{7}$ who claimed that phenobarbitone would prevent febrile convulsions if the plasma concentration was maintained at the high level of $69 \mu \mathrm{mol} / \mathrm{l}(16 \mu \mathrm{g} / \mathrm{ml})$ or over. As their trial included only a few patients and because of the importance of the conclusion for preventive paediatrics we repeated the study on a larger sample.

\section{Patients and methods}

Febrile convulsions were defined for the purpose of this study as convulsions occurring with fever due to infections other than those primarily affecting the brain. ${ }^{8}$ The incidence of repeat convulsions within six months of a first convulsion was determined in two groups of children aged six months to three years who presented at the Royal Hospital for Sick Children, Glasgow, from September 1973 to September 1974. The nature and purpose of this trial was explained to the parents, whose permission was essential to their child's inclusion in this study. In addition to those with central nervous system (CNS) infections, children were excluded if there was a history of previous convulsion or evidence from the history or examination of pre-existing CNS abnormality.

Acute medical care in the Royal Hospital for Sick Children is shared equally between two medical paediatric units. Children suitable for the trial attending the hospital were allocated into a treatment or control group according to which unit was "receiving" at the time of the convulsion. Thus children having a convulsion in the 24 hours starting at 9 am on Mondays, Wednesdays, Fridays, and alternate Sundays became controls and received no treatment while prophylactic phenobarbitone was given to those who presented on the other days.

There were no differences between the two medical units in the management of the presenting convulsion. Those children who were convulsing on admission to hospital were treated successfully with intravenous diazepam. High fever was reduced by tepid sponging. Children admitted into the treatment group were prescribed phenobarbitone $4-5 \mathrm{mg} \mathrm{kg}^{-1}$ day $^{-1}$ in divided doses. They were seen in a special clinic after enrolment in the trial and then every one to three months for six months. At each attendance venous blood was taken for phenobarbitone estimation and the dose adjusted if necessary to 